\title{
Composite Content Management-Case Management and Enterprise Content Management
}

\author{
Lekha Menon \\ HiTech Solutions Central, Tata Consultancy Services Limited, Mumbai, India
}

Received 2012-03-12, Revised 2012-07-24; Accepted 2012-08-29

\begin{abstract}
Interest in Composite Content Applications has been climbing higher over the recent years due to the rising need around enabling business process improvements and addressing challenges around business process agility. Such applications can deliver substantial value by leveraging both content and process services for the better orchestration of people and processes and empower organizations to automate routine tasks and aggregate information from multiple sources in a collaborative work environment that enables rapid decision making. In this whitepaper, we have introduced Case Management and its relationship with ECM, the essential functionalities in a Case Management Product stack and expanded on leading vendors in this space along with the salient features of their case management offerings. Subsequently we have built on a sample case scenario of building a contracts management application using EMC Documentum xCelerated Composition Platform (xCP), the business needs and challenges that a well-designed Contracts Management application can address, a real-life application of xCP platform to build such a solution and the observations on the strengths and limitations of this platform, along with a few workarounds to address the limitations.
\end{abstract}

Keywords: Enterprise Content Management, Case Management, Documentum xCelerated Composition Platform, Documentum xCP, Contracts Lifecycle Management

\section{INTRODUCTION}

Case Management has been a rising trend in the ECM landscape since the past couple of years. ECM vendors look to provide a more focused solution based on the underlying content management platform. Vendors have built products to solve business problems using an integrated platform that covers a broad array of capabilities, which include content management, document imaging, business process management, records management, business rules, analytics, collaboration and customer communication management. ECM products built on the case management foundations are much more agile and adaptive to the changing business needs of an organization than before.

\subsection{Introduction to Case Management}

Businesses in long-term relationships with their customers are expected to store accurate records, communicate with their clients in an effective, personalized and timely fashion and improve the speed, efficiency and accuracy of handling each case (AIIM, 2010). It is challenging to meet the business goals mainly due to the following factors:

- Customer mobility

- Channel-related factors such as multiple communication channels and multi-channel distribution needs

- Increasing personalization requirements

- Merging customer-related data that is usually present in distributed silos of information

- Compliance-related factors such as frequency of changes in products

- Today's jobs are less structured, more ad hoc and require more skills and interaction with other specialists

- There's greater emphasis on automating and tracking inconsistent "incidents" that do not follow a well-defined process 
- There are new demands that regulators, auditors and litigants place on business to respond to external regulations

- Demographic trends will create a shortage of skilled workers and the current workers are not "empowered"

Customer interfaces are handled in a CRM or helpdesk system, which holds activity histories of the customers, incoming and outgoing emails, letters and documents and manages multiple connected relationships between various participants such as customers, members, citizens, families, assessors and care professionals. However, the CRM and help-desk systems lack in their capability to group together such participants on a case-to-case basis.

Generally, teams that need to collaborate for any Case Management-related activity do so by using emails. However, emails are difficult to track and do not provide adequate security. Moreover, collaboration through emails may lead to duplicated efforts and result in silos of information. Additionally, emails do not necessarily conform to regulatory and compliance requirements.

\subsection{ECM for Effective Case Management}

ECM is the strategies, methods and tools used to capture, manage, store, preserve and deliver content and documents related to organizational processes. ECM tools and strategies allow the management of an organization's unstructured information, wherever that information exists.

ECM offers a secure, electronic repository for unstructured content that comes from various sources and in multiple formats. It can link this information to a record in another enterprise, departmental or industryspecific system for rapid retrieval. It can also send this content through a simple, linear workflow for business process management. Thus ECM can provide many benefits, including faster business processes, reduced document storage costs and enhanced information availability and security. But in today's times there is a continuous need to improve business agility by enabling transparent and agile processes and faster decision making capability to business users.

Case Management, at the most elemental level, is the electronic equivalent of what has supported business over several centuries: the cabinets, drawers, folders, forms and collaborative workflow processes supporting important human decisions. Case management captures the backward-and-forward nature of human interaction to reach a specific outcome. Case management departs from the traditional view of structured and sequential predefined processes. Instead, workflows are nondeterministic, meaning that they have one or more points where different continuations are possible. They are driven more by human decision making and content status than by other factors. Because the progression of work cannot be completely anticipated at design time, any solution needs to be able to accommodate more dynamic execution, in which decision and content state changes are used to trigger the workflow.

Any one case, contract or claim may involve more than one customer, knowledge worker or other contributor and entail the use of multiple communications channels between these participants such as web forms, letters, emails, faxes and outgoing print, SMS messages, instant messages, social forums and even Tweets. The case file itself will involve a collection of customer communications, forms, process documents, reports and supporting documentation and will need to be managed for compliance and audit. This may best be handled by an Enterprise Content Management (ECM) system, but the individual documents will need to be grouped together as part of the case file and made accessible in a suitably controlled way to all who are involved in the case, including those at the customer interface and a workflow or business process needs to take place to move the case to its outcome as shown in Fig. 1.

The concept behind case management is not new and several vendors have been providing case based solutions for several years using a combination of document management, workflows and imaging. However, recent trends and market pressures demand a more agile, efficient and collaborative mechanism for case management. ECM systems taking a singledocument view, rather than focusing on a case file, frequently require customization or procedural acrobatics to manage case-based scenarios. Case processing has very specific needs in terms of adaptive or dynamic workflows being able to accommodate ad hoc routings and rapid process changes. Cases may involve collaboration with others outside the organization and these relationships may vary case-by-case. Above all, case managers need to be able to monitor and manage progress as shown in Fig. 2.

\subsection{Composite Content Applications}

An ideal Composite Content Application needs to be simple and flexible, to enable business agility. Such and application should address the following characteristics (Roe, 2010):

- Standards-based and open repositories-for ease of integration across multiple content sources

- Content automatically integrated into processes-for effective business process management 


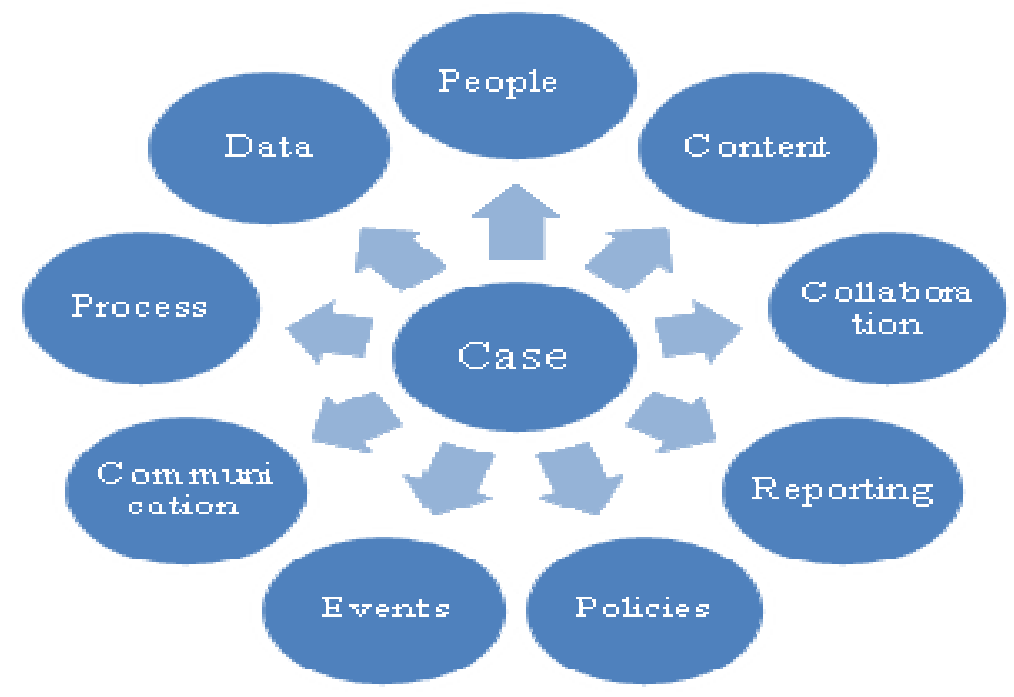

Fig. 1. What Constitutes a Case

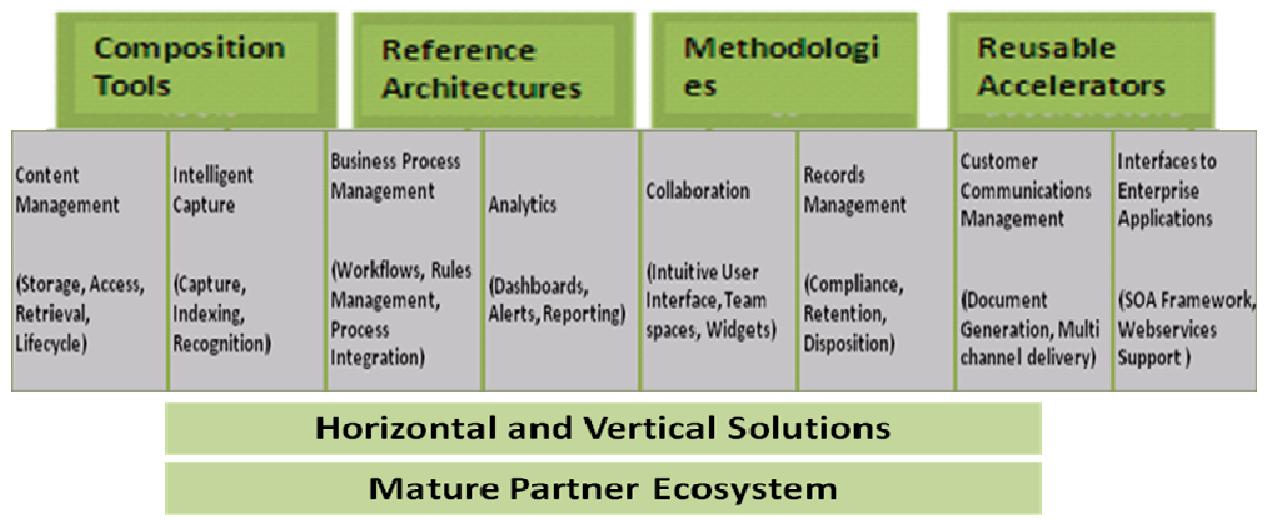

Fig. 2. Case Management Framework

- Powerful tools to derive intelligence from contentcontent analytics for real-time visibility

- Familiar, easy-to-use frameworks, attractive and flexible experiences for any business user-Businessuser-friendly dashboards, models, policies, rules and reporting

- Quick deployment without changing entire system

- Application interfaces to CRM, ERP, Supply Chain Management, Legacy and Web data

There are eight critical capabilities that can really help distinguish the right fit for enterprises based on how they intend to use the solutions. The critical capabilities for Composite Content Frameworks include the following (Gartner, 2012).
Balanced content, collaboration and process services within an SOA framework.

Content, collaboration and process have to be closely and seamlessly integrated, as well as the product stack should be designed as per Service Oriented Architecture (SOA) so that connecting to any application and data source can be done using standards-based SOA, using the right content and process components as callable services.

Integrates well with other critical content and process components.

Every content architecture includes repositories and application interfaces that will not be provided by any one suite vendor and there will be a need to integrate with content and process repositories of other vendors. Products 
that comply with standards such as CMIS (Content Management Interoperability Services) will provide an assurance to customers that their existing technology will be open to integrate or connect with any new technologies in future, with minimal custom code or adapters.

Intelligent and versatile on-ramps and off-ramps (document capture, optical character recognition, eforms and composition).

Case Management needs tools that can efficiently capture paper documents, images, voice messages as well as deliver content like reports, letters, statements to the end customers. Thus intelligent capture tools, imaging and indexing capabilities, e-forms and document automation features should be inherently supported by case management vendors.

Early focus on a core vertical or horizontal configuration via predefined case-specific data models, hierarchies and life cycles.

Some suite vendors have had substantial experience in specific configurations because their partners and their customers are specialists. Transferring the knowledge gained over several years of understanding customer requirements related to a particular domain or industry area and leveraging this knowledge in developing the Case Management platform will act as value added factors. There are also cases where service partners use a case management platform to build industry solutions on top of vendor-provided framework.

Application interfaces to CRM, ERP, supply chain management (SCM), legacy and Web data.

Some ECM and Business Process Management (BPM) suite vendors are certified partners of one particular ERP, CRM or other business application. Most are able to support standards-based integrations with other applications via Web services, enterprise service buses and so on. Easy integration capabilities with enterprise applications and legacy applications are essential features.

Comprehensive and highly configurable case-based user interface.

Shortage of skilled resources and increasing workloads essentially require that the time spent on training and moving to new interface is limited to create a better acceptance of the platform. The interface should hence be user intuitive and configurable such that users can leverage the Graphical user tools that enable Rapid app development without coding and ensure that changes can be incorporated quickly and easily.

Business-user-friendly dashboards, models, policies, rules and reporting.

Portals, Dashboards, Analytics and Mashups are some of the desirable aspects that will make a case management platform fare better. Any solution that involves the least amount of technology skill required for managing it, modifying it and getting meaningful information from it will score better.A platform with advanced analytics capabilities, simple administrative controls, Business Rules management, Business Activity monitoring to enable a business owner to gather appropriate and relevant feedback easily will be an important requirement.

Mature, certified and sustainable partner ecosystem.

Most vendors have limited domain expertise in building case management solutions is limited and depend heavily on end customers or system integrators to develop solutions on top of their framework. A mature and developed partner ecosystem will enable the development of predefined case templates, in-built processes and sample dashboards that will enable the platform to gather better acceptance from similar industry vendors.

There are a number of ECM and BPM vendors in the market providing such applications, a few of them being Appian, Pallas Athena, Pegasystems, Singularity IBM FileNet, Laserfiche, Nuxeo, Alfresco, EMC Documentum xCP, Case 360, Oracle Case Management.

\subsection{Case Management Vendors-Market Presence}

ECM vendors have historically excelled at providing 1st generation Case Management solutions as they specialized in the ability to capture content in an ad-hoc and unstructured manner and distribute that content ondemand to workers. This provided users with faster and easier access to data and content, but did not usually help a manager manage the rules, policies and efficiency in how cases are handled.

Similary, workflow software helped users route work items to appropriate people, but did not help process managers manage the efficiency and distribution of the process underlying the workflow. Many BPM vendors also support Case Management, but they often do not look at content in the same light as ECM systems.

A few leading Case Management vendors and the salient features of their products are listed below.

\subsection{Pegasystems}

- Over 30 packaged case applications across multiple verticals

- Particularly strong for the service request area

- Includes predictive analytics

- Domain expertise acquired through Chordiant Software 
- The underlying rules capability of its engine enables the incremental automation of the case flow

- Rules-based automation, routing and escalation

- Advanced, Event-Based Process Automation

- "Save as template" feature: Save ad hoc manual cases and tasks as templates to be applied in future, similar situations

\subsection{IBM}

- Strongest scores across the three use case categoriesinvestigative, service request and incident management

- $\quad$ Strong BPM, ECM and analytics

- $\quad$ Strategic and strong partnership ecosystem

- IBM FileNet Business Process Framework for case management was originally based on a unified platform that was a leader in both the ECM and BPMS markets

- Scalable platform, comprehensive control over content objects, records management, rich process tools, services orientation

- ECM widgets for a better content client

- Some weakness in collaboration and user experience

- Degree of difficulty in buying a "case management solution" from IBM has grown, as so many products and parts could apply.

\subsection{EMC}

- EMC Documentum xCelerated Composition Platform (xCP) is robust and features a good balance of content and process functionality

- The Documentum family includes core ECM and BPM suites and extends to integrated archiving, ediscovery and compliance (EMC SourceOne), secure extended enterprise collaboration (EMC CenterStage), intelligent capture (EMC Captiva), reusable user interface components (TaskSpace) and customer communications management (EMC Document Sciences)

- Collectively, these capabilities are exposed as services within the xCP platform and extend EMC's overall value proposition for case management solutions development

- Composition tools-Set of graphical tools that allow rapid application development with little or no use of coding. These tools empower business users to create applications through configuration, modeling and composition

- xCelerators-Set of reusable artifacts that improve speed of development and deployment of applications and solutions

\subsection{Appian}

- Appian provides a strong integrated offering

- Complete integrated suite solution with all the components necessary to build a rich case management application with either an on-premises or SaaS-based solution

- Well-balanced product with strong capabilities across design, development, reporting, content and specific case automation features

\subsection{Singularity}

- Singularity links business goals and processes, providing strong collaboration and mobility support for workers to personalize their desktops

- It offers a strong event model with clear product support for complex timelines and skills/sampling enhances the offering

- Particularly relevant for Microsoft shops, the Singularity product leverages Microsoft Office, SharePoint, Dynamics/CRM and OCS/Lync

\subsection{Global 360}

- Global 360's Case360 provides balanced content and process technology

- Marketing focus is on fast deployment, lower overall costs and reusable process logic

- Includes all the basic components-A virtual file system, process controls, collaboration, audit trail and a user interface to task management and status reporting

- Detailed understanding of the landscape of case management types

- Strong design, automation and event management

- Integrated suite designed to address the mix of complex unstructured and structured processes that organizations face

- Focus on content-centric case management applications

- Provides an innovative vision as to how processes are developed based on a "persona" approach that focuses on the needs of case workers and stakeholders and leveraging emergent design principles

- Limited partnerships to promote its message

- Doesn't directly market ECM capabilities and has thus been hidden from some buyers

- Its content and collaboration functionality is weaker than its process components 


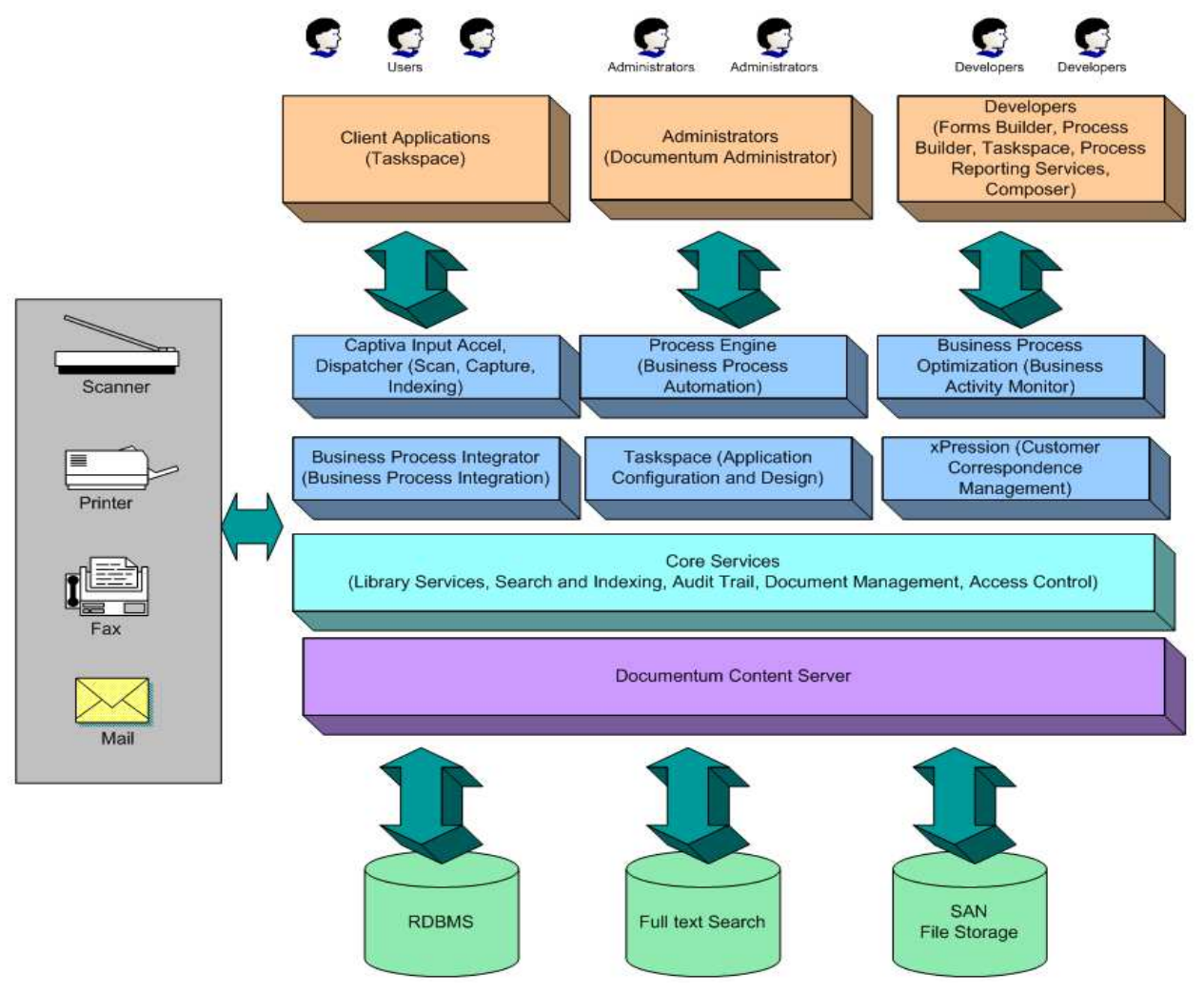

Fig. 3. $\mathrm{xCP}$ Architecture

The following sections expand on one of the above products-EMC Documentum xCelerated Composition Platform (xCP) platform in particular and its employment in building a sample case management application (in this case contracts lifecycle management).

\subsection{Documentum xCP}

Documentum $\mathrm{xCP}$ is a single, integrated platform that leverages Documentum ECM and BPM products to support the development of comprehensive case-based business solutions. As a composition platform, xCP provides reusable components, model-based configuration, a configurable user interface, dashboard tools and reference applications to enable rapid development.

$\mathrm{xCP}$ is a set of integrated products providing different functionalities. Documentum Forms Builder is the design tool that can be used to create web-based, graphical user interfaces to collect data from the users. These interfaces can be made available through

Documentum Taskspace is a highly configurable user interface that is closely and seamlessly integrated with Documentum Forms builder, Process builder, Business Activity monitor and the Content server. It thus unites the forms, processes, reporting and content into a single user experience.

The Documentum process builder is the business process design tool that can be used by business process users to create and configure workflows, by mapping it to the business processes. Designers can use the process builder to configure process activities, manual activities, automated tasks, work queues, automated content management actions and system integration logic using visual design techniques.

The Documentum business activity monitor and the process reporting services provide reporting and monitoring capabilities. The BAM taskspace component can be used as a part of taskspace and used to generate graphical dashboards to monitor the business processes. The documentum process engine automates all business processes created by the process builder and the process integrator manages the inbound and outbound integration of data and content. In addition, the Documentum Collaborative services provide a set of services and objects exposed via Taskspace to enable team collaboration activities and adhoc discussion threads on any taskspace item. 


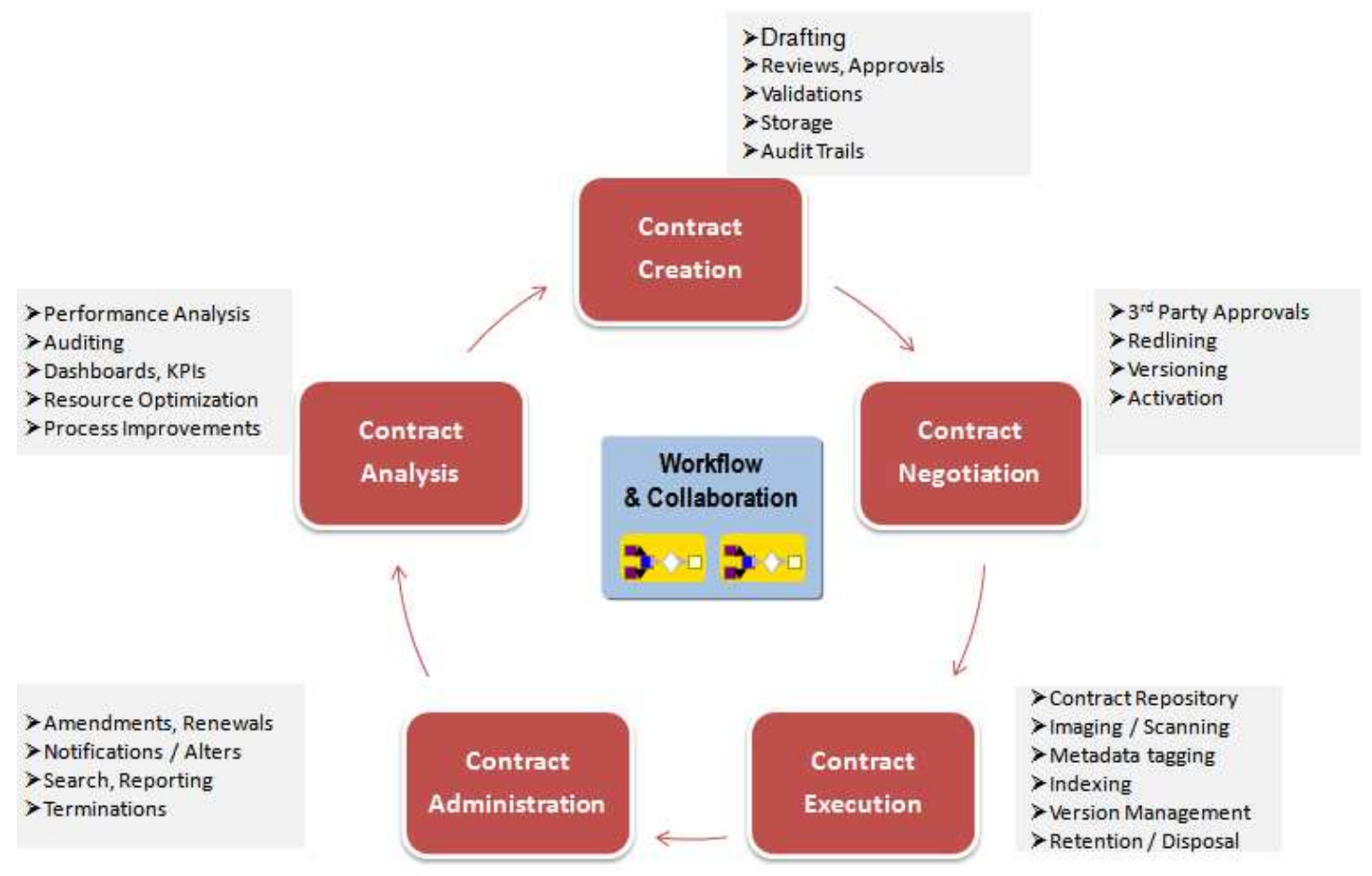

Fig. 4. Basic Components of a CLM Platform

Further, $\mathrm{xCP}$ includes a set of fully integrated technologies, with field tested development and deployment tools shown in Fig. 3. xCP also provides a set of xCelelerators that include process templates, blueprints, data structures, predefined integration points, best practices, design patterns, sample dashboards, express installers and sample applications. These xCelerators greatly reduce the time taken to develop any application in XCP.

\subsection{Contracts Lifecycle Management (CLM)}

Contracts are the foundation of any business and a typical company needs to manage thousands of active contracts that define business interactions among customers, vendors, partners and employees shown in Fig. 4. Contracts specify agreements related to everything from pricing to confidentiality and as legal instruments they carry the weight of litigation.

Contracts in paper format are challenging for many organizations to access and manage and digitizing them and storing them in an ECM system can provide wider employee access to the contract of record and time savings. Using ECM to support contract management leads to (PEGA, 2011).

\subsection{Reducing Paper and Storage Costs}

Contracts usually represent a voluminous amount of pages and using ECM can eliminate physical storage costs and vastly reduce the amount of paper exchanged.

\subsection{Providing Versioning and Audit Trails}

By using electronic contracts all parties benefit by having access to versioning and audit trails that can reveal discrepancies and aid understanding on the finer points.

Using workflow to manage milestones. Workflow rules around contracts can help flag accounts due for contract renewal or identify missing elements that need to be filled in for completion. Being able to modify workflows to support multiple contract types can also aid sales by offering alternatives for sales making contract changes on the fly to support changing conditions.

Contract lifecycle management (CLM) is the process of controlling, coordinating and streamlining all stages in the contract lifecycle (EMC, 2010).

\subsection{Central Contract Repository}

The foundation of a contract lifecycle management solution is a single, unified repository, which provides a 
web-based document management environment enabling full-text and keyword search of contracts and attachments. The repository should be able to manage faxed and scanned documents as well as native files in virtually any format, including all versions of the .doc, .pdf, .ppt and .xls file types and all the common image formats.

\subsection{Authoring and Negotiation}

A flexible authoring environment, seamlessly integrated with the contract repository, accelerates and controls the contract creation process and improves collaboration between buyers, sellers and their internal stakeholders. CLM contract authoring should featurerevision tracking, versioning, the ability to compare iterations side by side and a complete revision history, as well as access to a central library of preapproved clauses and terms that can be repurposed and shared across the enterprise. Contract documents can also be generated based on the already entered contract specific sections and clauses, which themselves can be derived from standard contract template as well as customer account specific clauses.

\subsection{Execution Management}

Process management provides a disciplined yet flexible means of control that orchestrates tasks, activities and decisions across the contract lifecycle. It brings standards and best practices to bear in contract approval, execution, tracking, enforcement and performance management. Workflows that employ approvals engage multiple departments simultaneously, each working solely on its portion of a contract. Graphical approval workflows display where a contract is in the approval process, who still needs to approve it and can even note the role and relevance of each approver. Advanced CLM solutions also support "dynamic approvals"-workflows that enable the approval chain to change automatically as components of the contract are modified, adding or removing steps and approvers. In the area of contract administration, process management functionality helps administrators track and enforce obligations and monitor key milestones via automated alerts triggered by business rules. These alerts reduce all manner of issues that contribute to revenue leakage and unrealized savings opportunities: missed payments, over and under payment, billing errors and renegotiation windows that pass during the contract renewal period.

\subsection{Administration}

Using new generation of search and discovery tools to locate existing contracts, amendments and renewals, extract key content from the contract documents and updating the associated metadata based on extracted content can improve the ease of finding relevant contracts in a quick and efficient manner, handle contracts renewals and terminations on a more proactive and planned fashion and thereby reduce the administration effort involved.

\subsection{Analytics}

Alerts and reports are a critical component of contract management systems. Proactive email based alerts, KPI dashboards or more detailed management reports act as early warning systems for contract managers and key business stakeholders who have a vested interest in ensuring that key milestones and obligations are properly tracked and managed.

\subsection{Benefits of a CLM System}

An efficient CLM system will cut contract cycle times by eliminating common bottlenecks in contract creation, negotiation and approval, boost the workforce productivity by automation and streamlining the workflows, improve visibility of contracts thus aiding decision makers to make accurate assessments and improve contract fidelity by the use of templates, ensure contracts compliance via triggers, alerts and advanced dashboards.

\subsection{CLM using $\mathrm{xCP}$}

This section outlines the process by which the Documentum xCP stack can be leveraged to build a sample CLM application. It outlines the Business Needs and Challenges that were required to be addressed using a CLM Solution, the implemented solution and the observations on the strengths and weaknesses of the xCP Platform.

\subsection{Business Needs and Challenges 1.23. Challenges}

Manual process of managing contracts, linking contracts to master agreements. Data stored in excel databases making it difficult to link documents to their relevant metadata.

Inefficient Search and retrieval, Lack of a single repository to store all contracts.

Sub-optimal transaction processing efficiency: high turnaround time, difficult to do amendments.

Lack of integrated contract management system: distributed workflow with multiple legacy systems, manual workarounds/notifications, insufficient downstream integration, Reliance on email to handle contracts approval. 
High cost of management of contract management process with low process efficiency, high systems maintenance and services cost, quality/rework costs.

Difficulty in coping with high volumes growth in business-staffing model and systems not geared to support high growth in small market business.

\subsection{Needs}

Build a custom application that is highly available and accurately maps client specific requirements with minimal effort.

Solution to have an enriched end user experience.

Map the business processing needs to the solution, Predefined workflow-approval process to ensure right people sign off on contract and alerts to manage the contract renewal cycle.

Centralized contract repository with ability to search and view contracts, Document management including creation, redlining/editing and version control, Ability to create, store, edit and manage various subcontract documents.

Agile Development and Short turnaround time.

\subsection{Features}

The basic features required in the application are given below:

\author{
Browse for Content \\ View Content \\ Contract Specific Attributes/Metadata \\ Document Relationships, Discussion Threads \\ Search (including Fulltext) \\ Do Review/Approval \\ Workflow Reporting \\ Contract Lifecycle
}

\subsection{Solution Implementation}

The following sections outlines the means using which the above features were implemented using the Documentum XCP stack of products.

\subsection{Contract Browsing, Viewing and Updating Attributes}

A form template was designed using forms builder, for users to browse and view contracts and to update the relevant properties.

The Documentum Forms Builder is an interactive tool that allows creating and modifying form-based templates and storing them in the documentum repository.
A folder contents template was used to show specific contract types and relevant metadata. Action buttons were added on the screen to do enable relevant actions based on user privilege. Users can import new contracts using the 'Import' button at the bottom of the screen. On right-clicking on a particular contract, users can do required operations on it, such as viewing and updating its properties/metadata, editing the contract, emailing it as a link to other users, start an approval workflow, initiate discussions on it, link one contract to another such as linking a Statement of Work contract, a work order or an amendment to its Master service agreement. These menu options can easily be configured to show only the relevant options based on the user's privileges and it requires no additional coding. This ensures that even nontechnical users can design the user interface based on business requirements.

\subsection{Contract Specific Attributes/Metadata}

A document view template was designed to view and update the properties or metadata of the selected contract. When a user selects a contract from the main screen and selects 'Edit', the contract opens up along with its associated metadata. The actual contract is displayed on the right side of the screen using the inbuilt document viewer of taskspace.

\subsection{Contracts Search (Including Fulltext)}

Search templates were designed to define the search parameters, search fields, search query. So as to enable users to search on contracts based on contract types, effective dates, partner names, expiration dates. The search results can easily be configured to be exported to. csv files for later analysis. Users can search on the basis of metadata as well as on content within the contract (full text search). The Documentum search engine-xPloreprovides search services for the Documentum platform and delivers a search and indexing capability for the Documentum repository.

\subsection{Contracts Review/Approval}

Each contract would pass through a pre-defined number of phases with different users (draft, internal review, external review). It needs to be approved in each before final execution. After a user approves or rejects the contract document, he/she can put a comment in that and system will automatically show that contract document is approved or rejected by the respective user. The Documentum Business Process management suite comprising of the process builder, process engine and the 
process integrator provides the platform to design the Contracts Review and Approval process.

\subsection{Contracts Reporting}

Reporting provides visibility into all contractual relationships across business units, with a complete, field-level audit trail for every contract. It also enables performance assessment and risk analysis while delivering role-based dashboard views tailored for contract administrators, line-of-business managers, executives and other stakeholders. For example, via charts and summary tables, dashboards can highlight key performance indicators such as contract cycle time and revenue per contract or summarize compliance and execution milestones. This provided value to operational performance and compliance and also reveals would-be problems in time to take remedial action and can significantly reduce the resources spent on audits.

\subsection{Observations on Top Strengths and Limitations \\ 1.33. Strengths}

Integrated Framework, Tools and xCelerators to speed development time. Enables Rapid Development due to high configurability and user intuitiveness of Taskspace.

Scalable Workflow Capabilities.

Virtual or Compound Document functionality ideal for Case Management Scenarios.

Seamlessly integrates with Document Capture and Document Generation products and thus automate the entire lifecycle starting from the capture of hard copies to final customer correspondence.

\subsection{Limitations and Workarounds}

Full text search does not work by default in Taskspace and requires customizations. Taskspace has limited flexibility in customization and limited documentation available to provide guidance on Taskspace customization. As a workaround, there are a few xCelerators developed by the extended EMC community which can be installed additionally which will enable the full text feature on taskspace.

Collaboration Module is not very mature and does not cover the entire spectrum that industry leading Collaboration product support. EMC provides a separate collaboration product called Centerstage, which can be considered if there are advanced Collaboration needs. Another option would be to use Microsoft SharePoint as the collaboration portal and use the Documentum connectors for SharePoint to enable integration between the two products.

Managing rules is a limitation of the Documentum Process Suite and needs integration with rules engine products like Corticon. There are xCelerators available that can enable integration with Corticon. However the Corticon licenses will need to be procured separately.

High Fidelity Forms lack in flexibility and usability.

Not an ideal choice for Integration-centric BPM opportunities. For such requirements, BPM based case management products such as Pegasystems, Appian, Case 360 could be considered.

Limited Mobility support as of date. Developers can build custom apps using the Documentum Foundation Services (DFS) to address specific functionality.

$\mathrm{xCP}$ on the Cloud is not yet available for developers to leverage and the licensing model is quite complex to understand. Developers have the option to implement a solution using $\mathrm{xCP}$ and port it on to any Cloud infrastructure such as Amazon EC2.

\subsection{Evaluation of $x C P$ as a Case Management Platform}

Content Management-Provides a very strong content management platform including capabilities to manage all content types, centralized repository and well defined access control mechanism.

Business Process Management-Provides a good BPM stack for business process design and automation, but lacks in terms of flexibility, integration and ease of use as compared to leading BPM tools.

Collaboration-Provides an intuitive and configurable user interface, but the collaborative capabilities are limited.

Analytics-Provides basic analytics module for business process reporting, but there is a lot of scope in improvement in terms of flexibility and ease of use.

Intelligent Capture-Very strong document capture functionalities supported via EMC Captiva stack of products.

Customer Communications Management-Customer communications management is supported via DocSciences xPression module which provides mature capabilities.

Compliance-Records Manager and Retention Policy Services offer compliance and archival support.

Integration with Enterprise Systems-Supports open standards, web services and SoA framework, but integration with other applications require a good amount of coding and technical skills. 
Partner Ecosystem and Domain Solutions-EMC has a very good partner ecosystem who have developed a good amount of horizontal and vertical solutions on the xCP stack (https://community.emc.com/docs/DOC12583).

Tools and Accelerators-The EMC Developer community (https://community.emc.com) offers a good amount of reusable tools and accelerators, design patterns, reference architectures and guides, best practices which can speed the development duration.

\section{CONCLUSION}

Composite Content Applications are an emerging category where people, process and content interface efficiently to meet business goals in a rapid and agile manner. Case management frameworks and industry solutions provide value for money and a better time to market for customers to address specific business needs.

An ECM platform designed to enable Composite Content Application development provides many benefits to businesses, including:

- Rapid Prototyping for Business Case developmentLeverage the foundations to rapidly build business solutions

- Accelerated timeframes enabling shortened projectsAlmost 50\% reduction in development effort

- Improved Decision making-Enables processes to be monitored in real-time to track KPIs, service level agreements and performance metrics, thereby aiding in process improvement

- Business User Empowerment-Enabling real-time decision making and thereby improving productivity

- Lower total cost of ownership

- Build Customer Brand Loyalty-Dynamic Customer Communications Management enables personalized customer communication and improving engagement

Businesses that require case management capabilities should take due consideration of the very specific requirements of case management when selecting a new or replacement ECM system. Creating a case file from multiple elements of content, linking case participants, flexible management of the workflow process and integrating with CRM or help-desk systems are important requirements if expected productivity gains are to be achieved. Enterprise business planners, enterprise architects and line-of-business leaders are advised to review the benefits of a reusable, vendorsupported case management configuration and score vendor offerings based on critical capabilities for the most likely usage scenarios.

Composite Content Management can enable businesses across various industry verticals to leverage the case management model to rapidly build processcentric, case-based solutions using a configuration rather than customization approach. This will enable business stakeholders to maximize time to value and realize faster Return on Investment (RoI), reduce the cost and deployment risk and create an agile and flexible application which is more conducive to adapt to rapid changes in business and impact organizational performance at various levels.

\section{REFERENCES}

AIIM, 2010. Adaptive case management-comparing document-centric and customer-centric approachesa. ISIS Papyrus America, Inc. http://www.isispapyrus.com/Download/whitepapers/ISIS-AIIMCase-Management-Whitepaper-2010.pdf

EMC, 2010. 15-Minute guide to contract lifecycle management. EMC Corporation. http://www.emc.com/collateral/software/15-minguide/h4824-15-min-contract-lifecycle-mgmt-gd.pdf

Gartner, 2012. Access has expired. Gartner, Inc. http://www.gartner.com/technology/mediaproducts/newsletters/expiration_page/expiration_pa ge.jsp

PEGA, 2011. The Forrester Wave ${ }^{\mathrm{TM}}$ : Dynamic Case Management Q1 2011. Pegasystems ${ }^{\circledR}$ Inc. http://www.pega.com/resources/the-forrester-wavedynamic-case-management-q1-2011

Roe, D., 2010. An agile enterprise cms--what's that? Simpler Media Group, Inc. http://www.cmswire.com/cms/informationmanagement/an-agile-enterprise-cms-whats-that007279.php 